\title{
PROCEDIMIENTO PARA LA CONSTRUCCIÓN DE ESCALAS DE VALORACIÓN SEGÚN LA TÉCNICA DE LIKERT
}

\author{
Héctor Hernández Valz
}

\begin{abstract}
RESUMEN
En este artículo se presenta sucintamente la técnica para elaborar escalas de valoración en las que se miden valores referidos a comportamientos específicos. El punto de partida es la comparación entre tres definiciones para ilustrar el carácter de sentimiento de los valores para optar al final por la definición de Rokeach, según la cual usa la técnica de Likert y cada ítem contiene la comparación entre el valor seleccionado y un valor competidor; atendiendo a la definición de Rokeach según la cual un valor es "una creencia relativamente permanente de que un modo de conducta particular o que un estado de existencia es personal y socialmente preferible a modos alternos de conducta y estados de existencia ". De está definición se ha lomado como criterio para la construcción de los itemes a la noción de preferencia. A continuación se presentan los pasos a dar desde el momento en que se eligen los valores que se van a medir.
\end{abstract}

Palabras clave: valor; item, tabla de especificaciones.

\begin{abstract}
In this article it is presented the technique succinctly to elaborate scales of valuation in those that values are measured referred to specific behaviours, The starting point is the comparison among three definitions lo illustrate the character of feeling of the values to opt at the end for the definition of Rokeach, according to which uses the technique of Likert and each article contains the comparison between the selected value and a value competitor, assisting lo the definition of Rokeach according to which a value is a relatively permanent belief That a way of particular behaviour or that an existence state is personal and socially preferable to alternating ways of behaviour and existence states». Of definition is he/she has taken as approach for the construction from the items to the preference notion. Next the steps are presented to give from the moment in That the values are chosen that you/they will measure.
\end{abstract}

Key Words: value, article, chart of specifications. 


\section{INTRODUCCIÓN}

La medición de valores es un tema especialmente complicado y sin embargo de gran importancia para el trabajo del psicólogo, tanto en los aspectos teóricos como en los aplicados.

Resulta particularmente difícil debido a que es un terreno en el que el psicólogo usualmente incursiona poco y como prueba de ello está el hecho de que es abordado principalmente por los estudiosos de la personalidad y por los psicólogos sociales. En el primer caso los valores tienen dos funciones, la primera "explica" la conducta puesto que es la última interpretación posible de una conducta, precedido por la actitud, como puede verse en el siguiente esquema:

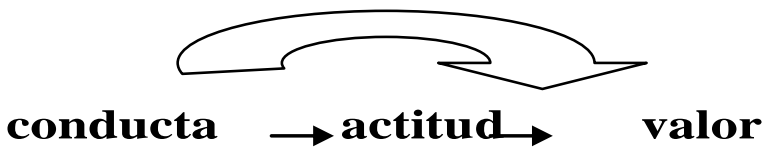

y es posible pasar directamente de la conducta al valor, lo cual es de suma importancia, pues posibilita la medición del valor debido a que la conducta se constituye como un indicador. Por su carácter de más o menos permanentes los valores constituyen un componente nato de la estructura de la personalidad. Por esta última razón se puede sostener que también tendrán una función de "justificador" de su conducta para el individuo, pues tiene la capacidad de permitirle el logro de su equilibrio psicológico. Los psicólogos sociales se basan en que los valores constituyen una especie de puente entre el individuo y el grupo, lo que queda de manifiesto en que explican el comportamiento de una colectividad apelando a las denominadas escalas de valores, y aquí utilizable únicamente como explicación.

\section{DEFINICIÓN DE VALOR}

No existe una definición única de los valores, y puede sentirse en cada definición la influencia de la disciplina del autor. Por ejemplo Kluckhon (1956) que es antropóloga se inclina por utilizar a los valores para explicar la forma en que cada cultura intenta responder las interrogantes que se desprenden de los problemas sociales actuales y considera que en cada cultura hay valores dominantes y valores variantes, los primeros corresponden al grupo dominante y los otros a los subgrupos sociales que se derivan del poder. La definición que emplea considera al valor como una preferencia que se cree y que se considera justificada, sea cual sea su origen o su campo de influencia.

Para Otaker Machotka (1964) el valor tiene un papel decisivo en la plena conciencia de sus actos, debido a que han pasado por el análisis en función de su moralidad.

Otro autor que ha hecho mucha investigación sobre valores es Rokeach (1972) quien considera al valor como "una creencia relativamente permanente de que un modo de conducta particular o que un estado de existencia es personal y socialmente preferible a modos alternos de conducta y estados de existencia"

Esta última definición en particular contiene el fundamento para la técnica de medición que se propone en el presente artículo, en tanto que afirma que "un modo de conducta particular o que un estado de existencia es personal y socialmente preferible a modos alternos de conducta y estados de existencia", Su función como principio para la 
técnica que se propone radica en que se transporta lo que ocurre en la vida diaria a una técnica de cuestio-ítemes deben reproducir con la mayor fidelidad posible la situación cotidiana de optar por una conducta frente a otra que para algunos individuos tiene diferente atracción, o para el mismo si las circunstancias varían.

\section{LA RELACIÓN ENTRE CONDUCTA, ACTITUD, INTERÉS Y VALOR}

El nexo que se encuentra entre las definiciones revisadas arriba es el de que los valores son creencias y como tales deben ser tratados como sentimientos y por tanto deben ser consideradas como medidas absolutas.

Con la finalidad de esclarecer los fundamentos de la medición de valores se hace referencia a los diferentes sentimientos y su relación con la conducta. La conducta se constituye como el elemento a partir del cual se infiere cualquiera de los sentimientos mencionados. En otras palabras, un sentimiento es la interpretación de una conducta, la cual puede ser ejecutada por el individuo que observa, realizada por una tercera persona o presentada en forma escrita $u$ oral.

Por otra parte se debe observar que dichos sentimientos están jerarquizados entre sí constituyendo el valor el nivel más alto y que, al ser interpretado sólo puede conducir a otro valor, en tanto que una actitud llevará a un valor pero no a otra actitud. Las actitudes y los intereses son sentimientos que pueden ser causales entre sí y ambos responder a un valor.

Pero lo más importante es que la medición de valores se ve facilitada por el hecho de que los valores pueden ser inferidos directamente de una conducta determinada, sin necesidad de que esta sea vista entes como actitud o como interés.

Para presentar la relación entre los diferentes sentimientos, actitud, interés y valor se hará uso del siguiente esquema

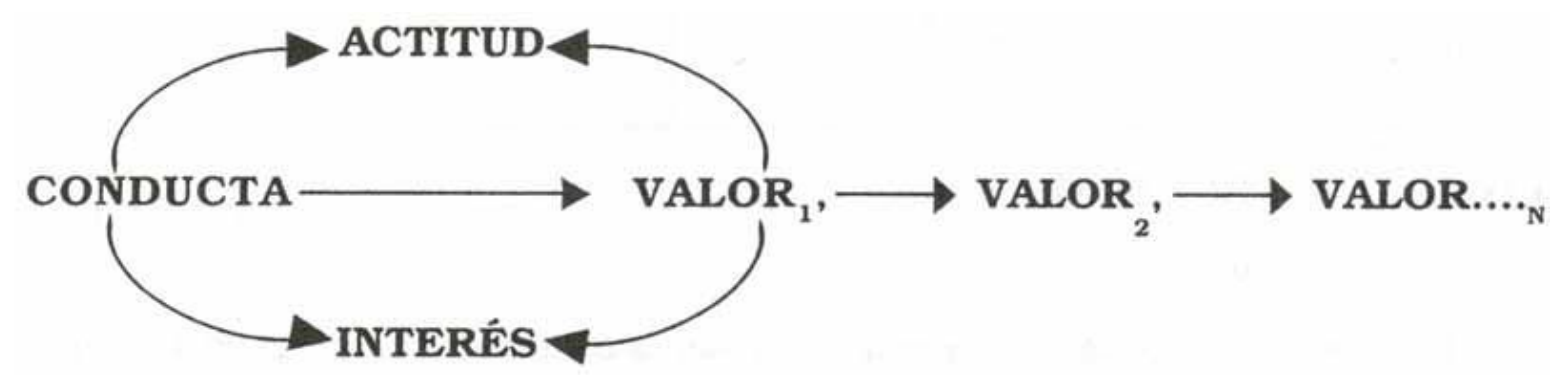

Las flechas sirven para señalar al nivel de interpretación inmediato

Procedimiento para la construcción de la escala de valoración según la técnica de Likert

Elaboración de la tabla de especificaciones

La tabla de especificaciones es la piedra angular para la elaboración de cualquier instrumento de medición debido a que es la síntesis del marco teórico que ha sido convenientemente desarrollado hasta el punto de contener los indicadores que serán utilizados para construir los ítemes. Pero además contiene la proporción de ítemes que corresponderá a cada dimensión del total de ítemes. 
Es una tabla de doble entrada con 6 columnas y tantas hileras como dimensiones se hayan elegido para construir el instrumento. La 1ra. columna es ocupada por la definición operacional del concepto con respecto al cual se han elegido los valores o dimensiones.

Para desarrollar el ejemplo se tomará el caso de la VIOLENCIA FAMILIAR

¿Qué es lo que se ve beneficiado o afectado si se efectúa la conducta $x$ ?

¿Para qué efectúa la conducta $x$ ?

Con la
Vísica
Violencia familiar $\left\{\begin{array}{c}\text { La integridad } \\ \text { El respeto } \\ \text { El amor }\end{array}\right.$

Una vez que se han respondido a la pregunta que sea pertinente es necesario seleccionar aquellos otros valores que marquen la dirección contraria y también capaces de atraer al individuo más que los valores que hemos señalado.

Ya establecidas las dimensiones el siguiente paso es redactar una lista de conductas que correspondan a los valores pertinentes al comportamiento referencial y a los valores competidores

\begin{tabular}{|l|l|l|l|l|c|}
\hline Comportamiento & $\begin{array}{l}\text { Dimensión } \\
\text { Valor medido }\end{array}$ & Indicador & $\begin{array}{l}\text { Valor } \\
\text { opuesto }\end{array}$ & Indicador & Peso \\
\hline Violencia familiar & $\begin{array}{l}\text { La integridad } \\
\text { física }\end{array}$ & $\begin{array}{l}\text { Cuidar la } \\
\text { salud }\end{array}$ & $\begin{array}{l}\text { Defender la } \\
\text { Traición }\end{array}$ & Usar la fuerza & 1 \\
\cline { 2 - 6 } $\begin{array}{l}\text { Cualquier acto dentro } \\
\text { del seno familiar con } \\
\text { implicaciones físicas, } \\
\text { psicológica, morales, } \\
\text { económicas, o sociales. }\end{array}$ & El respeto & $\begin{array}{l}\text { Escuchar } \\
\text { con atención }\end{array}$ & Mi imagen & Mostrar poder & 2 \\
\cline { 2 - 6 } & $\begin{array}{l}\text { Dedicar } \\
\text { tiempo }\end{array}$ & $\begin{array}{l}\text { Mi } \\
\text { conveniencia }\end{array}$ & $\begin{array}{l}\text { Lograr algún } \\
\text { Beneficio pers. }\end{array}$ & 3 \\
\hline
\end{tabular}

\section{REDACCIÓN DEL ITEM}

\section{Naturaleza del ítem}

El ítem de una escala de valoración según la técnica de Likert es una afirmación en la que se obliga al evaluado a comparar una conducta correspondiente a una de las dimensiones con otra conducta que es expresión del valor de dirección contraria

\section{ESTRUCTURA DEL ITEM}

De acuerdo con la descripción presentada en el punto anterior, la estructura del ítem puede ser esquematizada de la siguiente forma:

\begin{tabular}{|c|c|c|c|c|}
\hline $\mathbf{1}$ & $\mathbf{2}$ & $\mathbf{3}$ & $\mathbf{4}$ & $\mathbf{5}$ \\
\hline $\begin{array}{c}\text { Sujeto } \\
\text { Para mi }\end{array}$ & $\begin{array}{c}\text { Comparador } \\
\text { Es mejor mi } \\
\text { esposa }\end{array}$ & $\begin{array}{c}\text { Valor evaluado } \\
\text { Dialogar con }\end{array}$ & $\begin{array}{c}\text { Vínculo } \\
\text { que }\end{array}$ & $\begin{array}{c}\text { Valor competidor } \\
\text { Demostrar mi } \\
\text { autoridad }\end{array}$ \\
\hline
\end{tabular}


nario de lápiz y papel. Los

Sobre 1: El sujeto puede en primera, en tercera persona o tácito

Sobre 2: Indica al evaluado que está ante una comparación.

Sobre 3: Sirve de enlace contrastante entre los dos indicadores y varía de acuerdo con el comparador que se utilice.

Sobre 4: Es tomado de la tabla de especificaciones correspondiente a las dimensiones o valor evaluado. Ocupar el lugar 5 cuando se trata de hacer un ítem de dirección negativa o en contra del valor evaluado.

Sobre 5: Es tomado de la tabla de especificaciones correspondiente al valor competidor o negativo con respecto al valor evaluado. Ocupar el lugar 5 cuando se trata de hacer un ítem de dirección negativa o en contra del valor evaluado.

\section{LA CANTIDAD DE ÍTEMES}

El número total de ítemes está dado fundamentalmente por las medidas de validez y de confiabilidad, aunque son deseables las escalas más bien pequeñas que no fatiguen al evaluado.

\section{LOS GRADOS}

Se denomina con el término grados a la lista de categorías de acuerdo y desacuerdo que acompañan a cada ítem. La sugerencia principal es que se use un número par y que sea no menor de 4 ni mayor de 6 . aunque la teoría dice que pueden utilizarse hasta $10 \mathrm{u} 11$ grados

\section{EL ANÁLISIS ESTADÍSTICO \\ La Validez,}

La validez de estas escalas es de contenido y se obtiene por el Juicio de Expertos, debiéndose incluir sólo aquellos ítemes que tengan diferencia significativa al ser analizados con el Chi Cuadrado.

Una vez aplicada la prueba deben someterse todos los ítemes a la prueba de capacidad de discriminación, para ello se emplea la prueba t de Student, con la cual se comparan el grupo superior con el grupo inferior. Se aceptan sólo los ítemes que presentan diferencia significativa.

\section{LA CONFIABILIDAD}

El único método excluido es el de las dos mitades y por tanto se puede utilizar el análisis de consistencia interna utilizan la formula Kuder Richardson 20

$$
\begin{aligned}
\mathbf{K R}_{\mathbf{2 0}} & =\frac{\mathbf{K}}{\mathbf{K}-\mathbf{1}}\left(\mathbf{1}-\frac{\text { Sum Var }}{\text { Var Tot. }}\right) \\
\text { Donde } k & =\text { número de ítemes de la prueba }
\end{aligned}
$$

Otra alternativa la constituyen la de comparar con una prueba equivalente, aunque resulta difícil de construir una prueba similar y el test-retest, aplicable por el carácter más o menos permanente de los valores.

\section{BIBLIOGRAFÍA}

Rokeach. Milton. The nature of human values. New York : Free Press : London: Collier Macmillan Publishers. 1973. 